\title{
Novel Claw Pole Eddy Current Load for Testing DC Counter Rotating Motor - Part II: Design and Modeling
}

\author{
Khalil Kanzi *, Sam Roozbehani *, Abolfazl Dehafarin*and Majid Kanzi*
}

\begin{abstract}
Eddy current brakes are electromechanical devices used as variable mechanical loads for testing electrical machines. Accurate modeling of eddy current loss is an important $€$ factor for optimum design of eddy brake systems. In this second part, we propose novel formulations of eddy current loss in novel claw-pole eddy brake system. The proposed model for eddy current loss in novel claw-pole eddy brake system depends on the size of the claw poles. Also, in this paper, the flux density is measured by using the magnetic circuit of the novel claw pole. The model results are compared with experimental results and they are found to be in good agreement.
\end{abstract}

Keywords: Claw-pole eddy current brake, Counter-rotating motor, Load testing, Eddy current loss

\section{Introduction}

An eddy current brake is a new assistant brake system of large scale vehicles, e.g. modern high-speed trains, and plays an important role in the braking force induced within. We can expect economization, safety and a decrease in driver fatigue by using this type of brake [1]. If the coils shown in Fig. 1 are excited by a direct current and the disk is a solid iron body, eddy currents are induced in the iron by the relative motion between the disk and the magnetic field generated by the iron core. Interaction of the magnetic field and the induced eddy currents produces a retarding torque which is a function of the DC excitation current, rotor speed, and magnetic and electric properties of solid iron. Such a device can therefore be used as a load for testing the electric motor. The retarding torque of an eddy current brake is calculated as a function of the angular velocity of the disk and the DC current of the magnetizing coil [2].

In order to optimize the design of the brake system, it is necessary to estimate the braking force, the distribution of magnetic flux and eddy current loss. Many researchers have investigated much on the development of accurate measurement methods and analytical or numerical models for the eddy current force or eddy current loss. In [3]-[5], braking and attracting forces are calculated for different train speeds and excitation currents by using FEM. Finally,

\footnotetext{
* Iranian Academic Center For Education, Culture and Research

ACECR K.N Toosi University of Technology

Branch(Khkanzi@jdnasir.ac,ir, a_dehafarin@jdnasir.ac.ir)

Received 03 July 2012 ; Accepted 05 October 2012
}

numerical results are compared with measurements.

In [6], a new model is implemented in MATLAB to evaluate eddy current brakes. The model generates the characteristic curves of the brake being calculated from its configuration, dimensions and materials used. Also, in [7], a FEM method based approach is used to determine an eddy current brake model's parameters, i.e. equivalent resistance and reactance.

The present paper, together with a companion paper (Part I) [8], deals with the construction, design and modeling of the novel claw pole of the eddy current load constructed for testing counter-rotating motors. As shown in Fig.2, the construction of the testing system includes the counter-rotating motor (CRM) [9], eddy current loads and current sources.

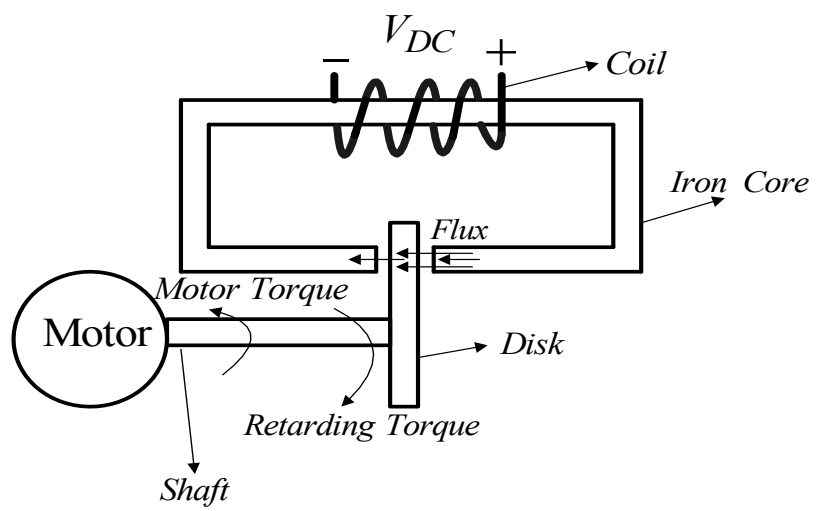

Fig.1. Schematic configuration of eddy current brake 


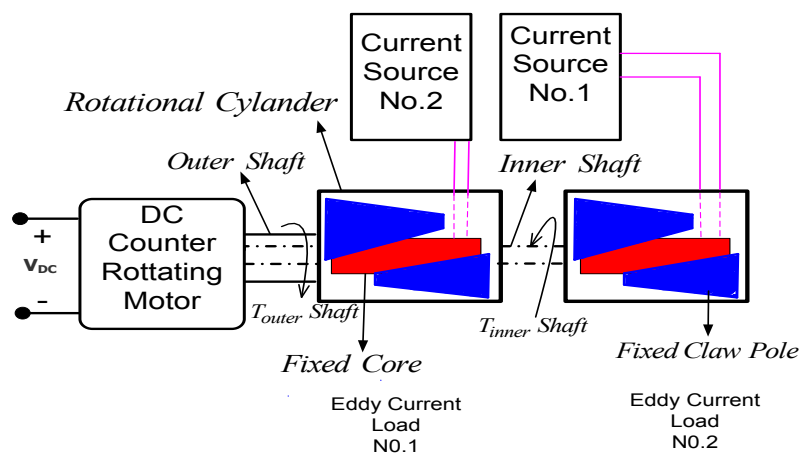

Fig.2. Schematic configuration of system

The CRM rotates in two directions simultaneously. So, two eddy brakes are employed as loads in this system. Each load is connected to either shaft of CRM. The stator of the novel load consists of only one excitation coil placed between some claw pole pairs. These claw poles are fixed and produce a magnetic field with a high number of pole pairs. As the current source is connected to the excitation coil, a magnetic field is generated in the core and the claw poles produce a magnetic field with a high number of pole pairs. In this state, as the cylinder is rotated by the DC CRM, an eddy current is induced within. Therefore, the mutual interaction of the eddy current and the magnetic field generates a retarding torque. Finally, the opposition between the retarding torque and the main torque (motor torque) causes heat loss in the cylinder. In this study, the eddy current brake modeling is done based on eddy current loss. According to the Steinmetz equation, the eddy current loss is as follows [10]:

$$
P_{e}=K_{e} \cdot B^{2} \cdot f^{2}
$$

where $f$ is the frequency of the external magnetic field, $B$ is the flux density and $k$ is a coefficient, which depe nds on the lamination thickness and conductivity of th e material, as well as other factors. In general, characteristics of the flux density and eddy current on the inner part of an electrical machine are investigated by using (1) and numerical analysis.

This paper presents the measurement and modeling of eddy current loss with various flux densities and speed vectors for the novel claw-pole eddy brake. The modeling of the eddy brake is done based on the size of the claw poles. In Section 2, formulation of eddy current loss for designing the novel claw-pole eddy brake is done, and then the method of flux measuring is presented in Section 3. Finally, a comparison of the calculations with the experiment results is done in Section 4.

\section{A formulation of eddy current loss for designing}

\section{the novel claw-pole eddy current load}

Eddy current loss occurs in a conductive material when it is subjected to a time-varying magnetic flux. In fact, the opposition of the retarding torque and main torque (motor torque) leads to heat loss in the cylinder. As the eddy current causes heat, this type of loss is called eddy current loss. Exact prediction of the eddy current loss in electrical machines is difficult. On the other hand, the value of the eddy current loss is an important issue, both in the designing and the analysis of eddy current brakes. In this section, the formulations of eddy current loss for the novel claw-pole eddy current load is done step-by-step.

\subsection{Geometric assumption}

The prototype eddy brake contains six claw poles. The calculation of eddy current loss is done only for one claw pole and then the result is generalized to six. In Fig.3, one of the claw poles is shown. This trapezoidal shape is divided into two parts. One part includes two rectangular regions and the other part includes two triangular regions. Because of the symmetry of the claw pole, the analysis is done only for half of it. In the rectangular part of the claw pole, the following equations are applicable:

$$
\begin{gathered}
\operatorname{tag} \theta=\frac{(b-a) / 2}{h} \\
\Delta x_{a b}=\frac{(b-a) / 2}{N} \\
x_{a b i}=\Delta x_{a b} * i \quad i=1: N \\
h_{a b i}=\frac{x_{a b i}}{\operatorname{tag} \theta}
\end{gathered}
$$

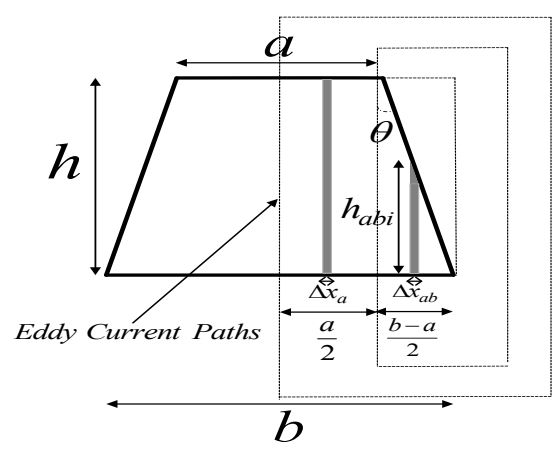

Fig.3. Claw pole

Also, for the triangular part of the claw pole, the following 
equations are assumed:

$$
\begin{gathered}
\Delta x_{a}=\frac{a}{N} \\
x_{a i}=\Delta x_{a} * i \quad i=1: N
\end{gathered}
$$

\subsection{Induced voltage}

As the current source is connected to the excitation coil, a magnetic field is generated in the core and the claw poles produce a magnetic field with a high number of pole pairs. In this state, as the cylinder is rotated by the DC CRM with a linear speed of $v$, a voltage is induced within the claw pole as follows:

$$
\begin{gathered}
e m f_{a b i}=B h_{a b i} v \quad: \text { for Triangle part } \\
e m f_{a i}=B h v \quad: \text { for Rectangular part }
\end{gathered}
$$

The value of the flux density $B$ in (8), (9) is determined in Section 3.

\subsection{The eddy current path:}

Eddy current is created by the induced voltage that is described in the previous section. Assumed eddy current paths are shown in Fig.3. The resistances of the paths are given by:

$$
\begin{gathered}
R_{a b i}=\rho_{f e} * \frac{l_{a b i}}{\Delta x_{a b} * T h_{D i s k}} \\
R_{a i}=\rho_{f e} * \frac{l_{a i}}{\Delta x_{a} * T h_{D i s k}}
\end{gathered}
$$

where $l_{a b i}$ and $l_{a}$ in (10) and (11) are:

$$
\begin{gathered}
l_{a b i}=4 x_{a b i}+2 h_{a b i}+4 x_{a b i} \\
l_{a}=4 x_{a i}+4\left(x_{a i}+\frac{b-a}{2}\right)+2 h
\end{gathered}
$$

\subsection{Eddy current losses:}

According to (8), (9), (10) and (11), the eddy current losses in either part of the claw pole, including the triangular part and the rectangular part, are obtained from the following relations:

$$
\begin{gathered}
P_{\text {lfeabi }}=\frac{e m f_{a b i}^{2}}{R_{a b i}} \\
P_{\text {lfeai }}=\frac{e m f_{a i}^{2}}{R_{a i}}
\end{gathered}
$$

The sum of the eddy current losses in the triangular and rectangular parts of the claw pole are as follows:

$$
P_{\text {lfe Traingle }}=2 * \sum_{i=1}^{N} P_{\text {Lfeabi }}
$$

$$
P_{\text {lfe Rectangular }}=2 * \sum_{i=1}^{N} P_{\text {Lfeai }}
$$

Finally, the total eddy current loss is given by:

$$
\begin{aligned}
P_{\text {ltotal }}= & 6 \times\left(P_{\text {lfe Traingle }}+P_{\text {lfe Rectangular }}\right) \times f\left(N_{r}, I\right) \\
& =P_{l}^{*} \times f\left(N_{r}, I\right)
\end{aligned}
$$

The coefficient " 6 " in (18) is used because the eddy brake contains six claw poles. Also, in (18), the nonlinear term of $f\left(N_{r}, I\right)$ is caused by asymmetric distribution of eddy current density on the surface. Here, $N_{r}$ is the rotational speed (rpm) of the eddy brake and $I$ is the excitation current (A) of the eddy brake. The description of $f\left(N_{r}, I\right)$ is presented in Section 4.3.

\section{Flux measuring by using magnetic circuits}

The flux length of the eddy brake is shown in Fig.4. According to the lengths shown, the reluctance of each part is calculated and the total reluctance is given by:

$$
\mathfrak{R}_{T}=\sum_{n=1}^{8} \Re_{i}=\sum_{n=1}^{8} \frac{l_{i}}{\mu_{i} A_{i}}=198988 \mathrm{AT} / \mathrm{Web}
$$

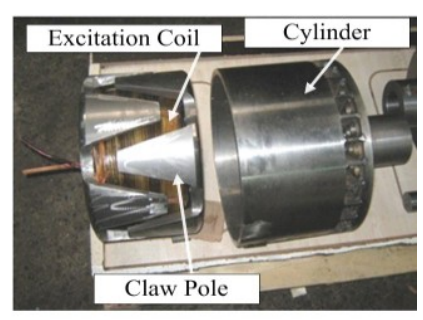

(a)

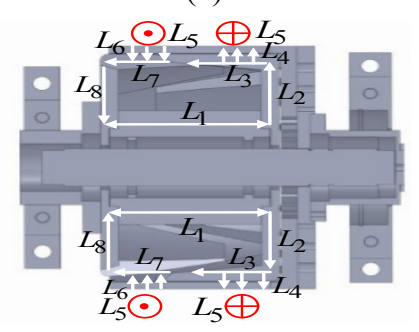

(b)

Fig.4. Prototype novel eddy brake. (a) Excitation coil, cylinder, claw pole; (b) Flux path in claw pole of eddy brake

Therefore, the flux in the air gap is given by: 


$$
\varphi=\frac{N^{\prime} . I}{\mathfrak{R}_{T}}
$$

where $I(\mathrm{~A})$ is the value of the excitation current and $N$ is the number of turns of the excitation coil. In the next step, the flux density that passes in each claw pole is given by:

$$
B=\frac{\varphi}{6 A}
$$

\section{Comparison of calculated and experimental results}

In this section, the results of the eddy brake model analysis and the results of the practical test of the eddy brake, for calculation of the eddy current losses, are compared by using Matlab/Simulink. Evaluating each method, the eddy brake losses are calculated for different speeds and for specified excitation currents. The experimental system is shown in Fig.5. The CRM is coupled to both of the eddy brakes. One of the eddy brakes is coupled to the inner shaft and the other to the outer shaft of the motor. Also, both eddy current brakes are connected to the current sources. The nominal current of these sources is $30 \mathrm{~A}$.

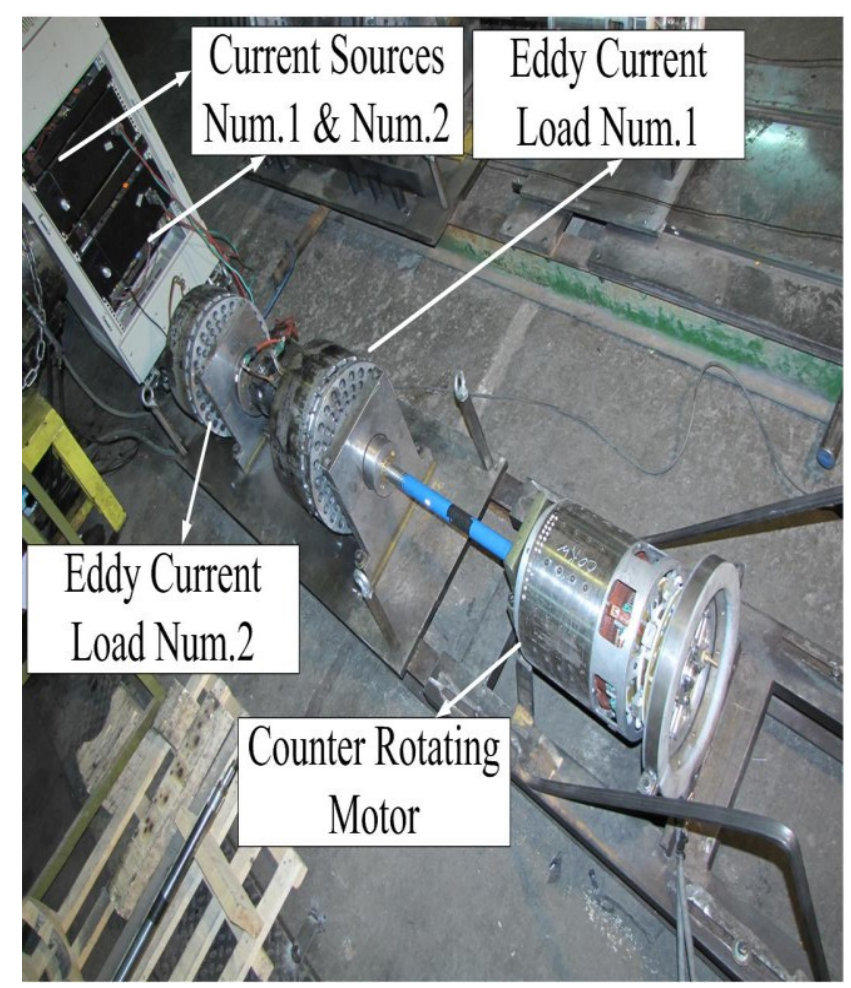

Fig.5. The experimental system

Since the two rotors of the CRM rotate in two opposite directions at the same time, the two eddy brakes do the same.Hence, testing of the motor is done in two states; in each, one of the brakes is blocked.

\subsection{Blocked eddy current no.2:}

In this state, the second eddy brake is blocked. Firstly, the motor is tested under no-load condition for which the excitation currents of the eddy brakes are set to zero. Hence, the eddy current loss of the eddy brake is assumed to be zero. Under this condition, the no-load loss of the system, which includes mechanical loss of the motor, eddy current loss of the motor, and mechanical loss of the eddy brake, is obtained by subtracting the input power of the motor from the copper loss of the motor.

In the next step, while the second eddy brake is blocked, the excitation current of eddy current brake no. 1 is set to a non-zero value. The loading loss of the system includes the mechanical loss of the motor, eddy current loss of the motor, and mechanical loss of the eddy brake. The eddy current loss of the eddy brake is obtained by subtracting the input power of the motor from the copper loss of the motor.

Finally, assuming that the sum of mechanical loss of the motor, mechanical loss of the eddy brake and the eddy current loss of the motor is equal in loading and no-load tests under the same speed conditions, the eddy current loss of the eddy brake is obtained by subtracting the no-load loss of the system from the loading loss of the system [8].

For example, the experimental test results of the motor for different values of voltage and speed, by setting the first current source to $15 \mathrm{~A}$, are tabulated in Table 1. In the sixth column of this table, the eddy current loss is calculated based on the measuring method that is described in the previous paragraph. Also, the eddy current loss in the fifth column of the table is calculated by using the novel formulation of eddy current loss in (18). As a comparison between these results, they are in good agreement with each other.

According to these results and by the interpolation method, the measured and calculated eddy current losses versus speed are shown in Fig.6. The flux of the eddy brake for $15 \mathrm{~A}$ excitation current is $0.0786 \mathrm{~T}$.

For specified values of current source, performing the same test to calculate the eddy current loss is done. For another example, the measured and calculated eddy current losses versus speed are shown in Fig.7 for 5 A excitation current. The flux of the eddy brake for 5 A excitation current is $0.0262 \mathrm{~T}$. 
Table 1. Measured and calculated eddy current losses in state 1

\begin{tabular}{|ll|ccccc|}
\hline$N_{r}(\mathrm{RPM})$ & $\mathrm{N}_{\mathrm{s}}(\mathrm{RPM})$ & $\begin{array}{c}\text { Current Source } \\
\text { No.1 }(\mathrm{A})\end{array}$ & $\begin{array}{c}\text { Current Source } \\
\text { No.2 (A) }\end{array}$ & $\begin{array}{c}\text { Calculated } \\
\text { Eddy Current } \\
\text { Loss (kW) }\end{array}$ & $\begin{array}{c}\text { Measured } \\
\text { Eddy Current } \\
\text { Loss (kW) }\end{array}$ \\
\hline 231 & 0 & 15 & 0 & 0.46 & 0.37 \\
495 & 0 & 15 & 0 & 1.930 & 1.789 \\
759 & 0 & 15 & 0 & 3.939 & 3.781 \\
1023 & 0 & 15 & 0 & 5.783 & 5.283 \\
1221 & 0 & 15 & 0 & 6.533 & 6.286 \\
1419 & 0 & 15 & 0 & 6.219 & 7.233 \\
\hline
\end{tabular}

Table 2. Measured and calculated eddy current losses in state 2

\begin{tabular}{|lcccc|c|}
$\mathbf{N}_{\mathbf{r}}$ (RPM) & $\mathbf{N}_{\mathbf{s}}$ (RPM) & $\begin{array}{c}\text { Current Source } \\
\text { No.1 (A) }\end{array}$ & $\begin{array}{c}\text { Current Source } \\
\text { No.2 (A) }\end{array}$ & $\begin{array}{c}\text { Calculated } \\
\text { Eddy Current } \\
\text { Loss (kW) }\end{array}$ & $\begin{array}{c}\text { Measured } \\
\text { Eddy Current } \\
\text { Loss (kW) }\end{array}$ \\
\hline 0 & 231 & 0 & 5 & 0.22 & .3011 \\
0 & 495 & 0 & 5 & .8415 & 1.022 \\
0 & 759 & 0 & 5 & 1.657 & 1.650 \\
0 & 1023 & 0 & 5 & 2.510 & 2.342 \\
0 & 1221 & 0 & 5 & 3.118 & 2.919 \\
0 & 1419 & 0 & 5 & 3.682 & 3.483 \\
\hline
\end{tabular}

\subsection{Blocked eddy current no.1:}

In this state, the first eddy brake is blocked and the stages in Section 4.1 are repeated. Regarding this point, the results of testing the motor under this condition are presented in Table 2. Also, the measured and calculated eddy current losses versus speed are shown in Fig.8 for 5A excitation current.

\subsection{Determining $f\left(N_{r}, I\right)$ :}

For determining $f\left(N_{r}, I\right)$, we used the surface fitting method. The measured results of the eddy current loss and the results found from (18) are only slightly different. The difference between these values arises from the asymmetric distribution of the flux on the surface of the claw pole. By calculating the ratio of the measured results of the eddy current loss to specified values of the current source, the formulation of this ratio is verified by fitting the surface and is given by:

$$
\begin{aligned}
& f\left(N_{r}, I\right)=10.08-0.001884 N_{r}-4.481 \times 10^{-7} \times \omega_{r}{ }^{2} \\
& -0.7201 I_{r}+\left(9.905 \times 10^{-5} \times \omega_{r} \times I_{r}\right)+\left(0.01305 I_{r}{ }^{2}\right)
\end{aligned}
$$

where $N_{r}$ is the speed (rpm) of the rotational cylinder of the eddy brake and $I$ is the excitation current (A). The surface of $f\left(N_{r}, I\right)$ for different values of speed and current is shown in Fig.9.

\subsection{Comparison of calculated and experimental results}

The accuracy of the formulation is assessed by an error estimator as [10]:

$$
E r r=\frac{\sqrt{\frac{1}{N-1} \sum_{i=1}^{N}\left(P_{i, \text { measurment }}-P_{i, \text { cal }}\right)^{2}}}{\max \left(P_{i, \text { measurment }}\right)}
$$

where $N$ is the number of measured data and are the measured and calculated core losses, respectively, and is a function applied to find the maximum element in an array. The values of error for specified values of current source and flux density are tabulated in Table 3. According to this table, the error has acceptable value.

Table 3. Comparison of calculated and experimental

\begin{tabular}{|c|c|c|c|c|c|}
\hline results & 5 & 10 & 15 & 20 & 25 \\
\hline $\begin{array}{c}\text { Ir } \\
\text { Flux } \\
\text { density (T) }\end{array}$ & 0.0262 & 0.0524 & 0.0786 & 0.1048 & 0.1310 \\
\hline Error (\%) & 0.7398 & 0.3964 & 0.6181 & 0.2933 & 0.2882 \\
& & & & & \\
\hline
\end{tabular}




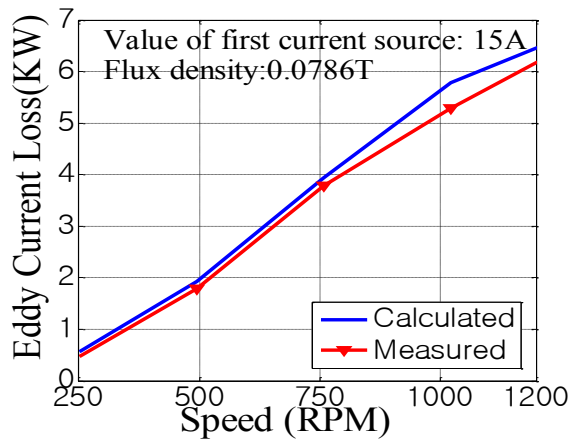

Fig.6. Eddy current loss for measured and calculated method (eddy brake no. 2 is blocked)

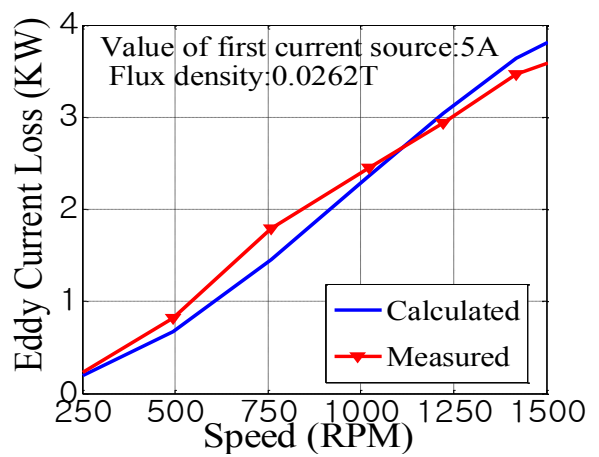

Fig.7. Eddy current loss for measured and calculated method (eddy brake no.2 is blocked)

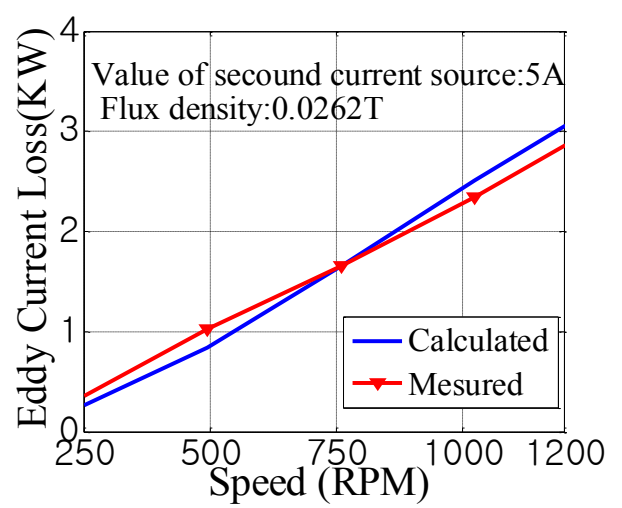

Fig.8. Eddy current loss for measured and calculated method (eddy brake no. 1 is blocked)

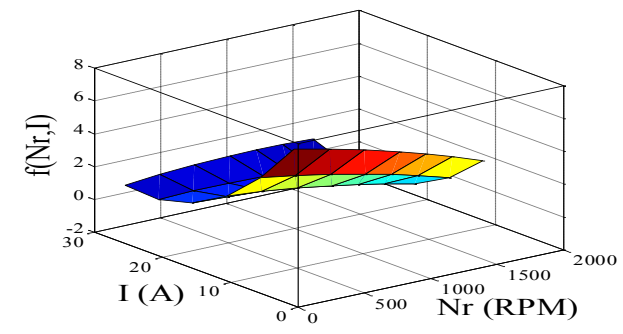

Fig.9. The surface of $f\left(N_{r}, I\right)$ for different values of speed and current

\section{Conclusion}

Providing a variable load for testing a motor under high speed conditions is usually a difficult task. The eddy current brake can be applied in order to provide a load for testing of motors. Since accurate modeling of eddy current loss is an important issue for optimum design of eddy brake systems, this paper deals with the formulation of eddy current loss within a claw-pole eddy brake. The proposed model for eddy current loss in the novel clawpole eddy brake shows its high dependency on the size of the claw poles. In addition, the results of calculation and measurement of the eddy current loss are in good agreement with each other. Therefore, the error has acceptable value. The remaining discrepancy between the measured and calculated values in the claw-pole eddy brake may have arisen from asymmetric distribution of eddy current density on the surface of the claw pole.

\section{References}

[1] Y. Um, S. Torii, D. Ebihara, I. Sanjo, Y. Tani, "Characteristic of Eddy Current on the secondary of Eddy Current Brake," in Proc. Electric Machines and Drive, 1997, pp. MC1/11.1 - MC1/11.3.

[2] D. Gonen, S. Stricker, "Analysis of Eddy Current Brake," IEEE Trans. Power Apparatus and System, vol. 84, pp. 357361, May 1965.

[3] M. Hofmann, T. Werle, R. Pfeiffer, and A. Binder, "2D and 3D Numerical Field Computation of Eddy Current Brakes for Traction," IEEE Trans. Magnetics, vol. 36(4), pp. 17581763, Jul. 2000.

[4] M. Fujita, T. Tokumasu, T. Yamada, "3-Dimensional Electromagnetic Analysis and Design of an Eddy Current Rail Brake System," IEEE Trans. Magnetics, vol. 34(5), pp. 3548-3551, Sept. 1998.

[5] D. Rodger, H. C. Lai, P. K. Vong, "Finite Element Models of Eddy Current Brakes," presented at the $2^{\text {nd }}$ Int. Conf. Computation on Electromagnetic, UK, 2002.

[6] B. Dietrich, I. E. Chabu, J. R. Cardoso, "Eddy Current Brake Analysis Using Analytic and FEM Calculations Part I: theory," in Proc. IEMDC, 2001, pp. 454-457.

[7] Danila, F. Sisak, S. Moraru, and L. Perniu, "Computer Aided Design by FEM Method For Eddy Current Brakes," in Proc. IEMDC, 2007, pp. 347-352.

[8] K. Kanzi, A. Dehafarin, S. Roozbehani, M. Kanzi, Q. Vasheghani, "Novel Claw Pole Eddy Current Load for Testing DC Counter Rotating Motor - Part I: Construction," Journal of International Conference on Electrical Machines and Systems, vol. 1(3), pp. 57-65, Sept. 2012.

[9] K. Kanzi, "Design and Implementation of a Counter Rotating DC Motor Rating 60 kW", Iranian Academic Center for Education, Culture andResearch (ACECR), K. N. Toosi University of Technology branch, Tech. Rep. (178621), Feb. 2011.

[10] J. G. Zhu, V. S. Ramsden, "Improved Formulations for Rotational Core Losses in Rotating Electrical Machines", 
IEEE Trans. Magnetics, vol. 34(4), pp. 2234-2242, Jul. 1998

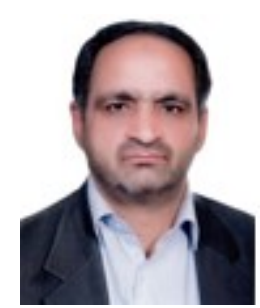

Khalil Kanzi received the B.S degree from K.N.Toosi University and M.Sc from Tarbiat Modaress University of Teheran in 1984 and 1992, respectively. He has worked for the ACECR Research Institute (K.N.Toosi University of Technology branch) in Teheran in the area of LV/HV high-power electrical machines (design and realization), power electronics and power systems for more than 20 years. He received the Ph.D. degree from K. N. Toosi University in 2009. He is now working at the ACECR Institute and his working area is design and realization of conducted EMI filters, electrical machines and FACTS controllers.

Sam Roozbehani received B.S and M.S degrees in electrical engineering in 2007 and 2011, respectively. He

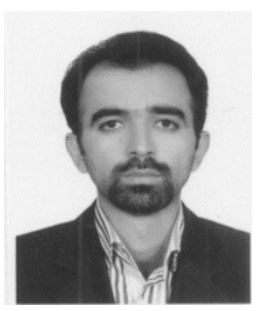
worked at Niroo Research Institute (NRI) in 2009. He is currently working as a researcher at the Academic Center for Education, Culture and ResearchK.N. Toosi University of Technology branch (Tehran, Iran). He is also a visiting instructor at Adiban Institute of Higher Education (University of Garmsar). His current research interests include renewable energy, wind turbines, application of power electronics in wind turbines, and drives of electrical machines.

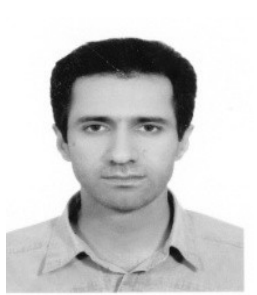

Abolfazl Dehafarin received the B.S degree from K. N. Toosi University of Technology in 1993. He has worked for the ACECR Research Institute (K.N. Toosi University of Technology branch) since 1995. His current research interests include design and simulation of electrical machines.

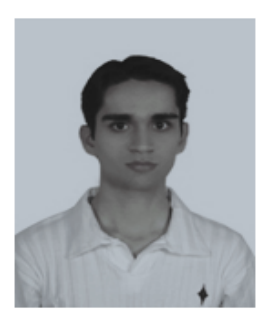

Majid Kanzi received the B.Sc. degree in Electrical Engineering from Islamic Azad University of Tehran and M.Sc. degree in Power Electronics at Kurdistan University of Iran in 2006 and 2010, respectively. Since 2004, he has worked on power quality in the electrical power network and from 2009 on improving power factors and eliminating EMI noise in the switching power supply. Also, he has worked on design and fabrication of electro-mechanical devices, such as the eddy current brake and DC counter-rotating motors at the ACECR. 